\title{
In-and-Out Molecular Changes Linked to the Type 2 Diabetes Remission after Bariatric Surgery: An Influence of Gut Microbes on Mitochondria Metabolism
}

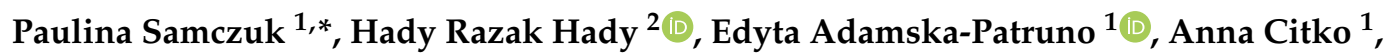 \\ Jacek Dadan $^{2}$, Coral Barbas ${ }^{3}$, Adam Kretowski ${ }^{1,4}$ and Michal Ciborowski ${ }^{1}$ (D) \\ 1 Clinical Research Centre, Medical University of Bialystok, 15-276 Bialystok, Poland; \\ edyta.adamska@umb.edu.pl (E.A.-P.); ankacitko@gmail.com (A.C.); adamkretowski@wp.pl (A.K.); \\ michal.ciborowski@umb.edu.pl (M.C.) \\ 2 1st Clinical Department of General and Endocrine Surgery, Medical University of Bialystok, 15-276 Bialystok, \\ Poland; hadyrazakh@wp.pl (H.R.H.); klchirog@umb.edu.pl (J.D.) \\ 3 Center for Metabolomics and Bioanalysis (CEMBIO), Universidad CEU-San Pablo, km 0, \\ Urbanización Montepríncipe. M-501, Alcorcón, 28925 Madrid, Spain; cbarbas@ceu.es \\ 4 Department of Endocrinology, Diabetology and Internal Medicine, Medical University of Bialystok, \\ 15-276 Bialystok, Poland \\ * $\quad$ Correspondence: paulina.samczuk@umb.edu.pl; Tel.: +48-85-746-88-94
}

Received: 3 November 2018; Accepted: 15 November 2018; Published: 24 November 2018

\begin{abstract}
Different kinds of gastrointestinal tract modulations known as "bariatric surgery" are actually the most effective treatment for obesity and associated co-morbidities, such as type 2 diabetes (T2DM). The potential causes of those effects have yet to be explained. In our study, we focused on molecular changes evoked by laparoscopic sleeve gastrectomy leading to T2DM remission. Two complementary metabolomics techniques, namely, liquid chromatography coupled with mass spectrometry (LC-MS) and gas chromatography mass spectrometry (GC-MS), were used to study those effects in a group of 20 obese patients with T2DM selected from a cohort of 372 obese individuals who underwent bariatric surgery and did not receive anti-diabetic treatment afterward. Modified levels of carnitines, lipids, amino acids (including BCAA) and $\alpha$ - and $\beta$-hydroxybutyric acids were detected. Presented alterations suggest a major role of mitochondria activity in T2DM remission process. Moreover, some of the observed metabolites suggest that changes in gut microbiota composition may also correlate with the tempo of diabetes recovery. Additional analyses confirmed a relationship between biochemical and clinical parameters and the aforementioned metabolites, thereby, highlighting a role of mitochondria and microbes. Our data suggests that there is a previously undescribed relationship between mitochondria and gut microbiota, which changes after the bariatric surgery. More investigations are needed to confirm and explore the observed findings.
\end{abstract}

Keywords: bariatric surgery; metabolomics; diabetes remission; laparoscopic sleeve gastrectomy; LC-MS; GC-MS

\section{Introduction}

Type 2 diabetes (T2DM) remission after bariatric surgery is a fact. Thus, gastrointestinal metabolic surgery is actually the most effective treatment for obesity and associated co-morbidities, like T2DM [1-3].

Bariatric surgery refers to three parts of anatomical gastrointestinal tract modulations-gastric restriction, exclusion of duodenum and upper intestine, and rapid delivery of food to the distal 
intestine or short common channel. These anatomical changes may induce a lot of physiologic and molecular changes helping to resolve type 2 diabetes [2]. The proposed mechanisms underlying diabetes remission after metabolic surgery included the starvation-followed-by weight-loss hypothesis, the ghrelin hypothesis, the lower intestinal (hind-gut) hypothesis and the upper intestinal (fore-gut) hypothesis. More theories have been proposed recently, including changes in bile acid metabolism and intestinal microbiome. Interestingly, none of these theories necessarily negate the others. These modulations result in reduced hepatic glucose, increased tissue glucose uptake, improved insulin sensitivity and enhanced $\beta$-cell function $[1,2,4,5]$.

Additionally, a discussion remains on which factors can play an essential role in predicting the impact of bariatric surgery on T2DM. Age, BMI, C-peptide, duration of the disease (ABCD score), $\mathrm{HbA1c}$, fasting blood glucose, incretins glucagon-like peptide-1 (GLP-1) and gastric inhibitory peptide (GIP)] can be included. Another example of a score grading system that is used to predict T2DM remission after bariatric surgery is the DiaRem score. It includes factors such as age, HbA1c, antidiabetic drugs and insulin, which are used to predict the remission T2DM. However, the DiaRem also has some limitations, e.g., the duration of T2DM omission [6]. Therefore, T2DM remission is probably an effect of a combination of a few mechanisms [7] and it can be difficult to design a study to elucidate a single mechanism. Fortunately, nowadays, we own research tools that allow the following of even the smallest modifications of numerous molecular factors in a single analysis, i.e., the "omics" sciences-genomics, transcriptomics, proteomics and metabolomics.

The potential of metabolomics in clinical research is well established. This includes studies related to diabetes, obesity and different aspects of bariatric procedures. Roux-en-Y gastric bypass (RYGB) and sleeve gastrectomy are the most frequently examined procedures; these have been routinely studied using metabolomics platforms such as liquid chromatography coupled with mass spectrometry (LC-MS) and/or hydrogen nuclear magnetic resonance $\left({ }^{1} \mathrm{H}-\mathrm{NMR}\right)$. Currently, most of the studies are focused on human or mice/rats samples with serum or plasma as the most commonly used biological materials. Although the influence of bariatric surgery on morbid obesity and type 2 diabetes were investigated the most frequently, other obesity-related diseases and health conditions were also occasionally studied [8].

While laparoscopic sleeve gastrectomy (LSG) along with RYGB are nowadays considered the "gold standard" of bariatric procedures [8,9], sleeve gastrectomy has become one of the most commonly used primary bariatric procedures for morbid obesity [10]. Moreover, LSG leads to significant improvement in biochemical glucose homeostasis and may be considered as a method for T2DM treatment [10]. Although molecular background of mechanisms underlying type 2 diabetes remission after bariatric surgery has been heavily investigated, it remains intriguing and has yet to be well defined $[2,8,11]$. Consequently, the aim of this study was to apply two complementary metabolomics platforms to investigate molecular changes accompanying early T2DM remission after LSG procedure.

\section{Results}

In the present study, accurate clinical, biochemical and metabolic data were analyzed to search for pre-operative and post-operative indicators of a higher remission tempo of T2DM after bariatric surgery. To recognize patients with quicker and slower rates of T2DM remission, a level of post-surgery HOMA-IR reduction was used. Three months HOMA-IR reduction has been previously proposed as the main predictor of T2DM complete remission [12]. Twenty obese patients with type 2 diabetes who underwent laparoscopic sleeve gastrectomy were included in the presented study. The selected study participants were divided into two groups (quicker $(n=11)$ and slower $(n=9)$ T2DM remission), depending on the rate of HOMA-IR reduction. The reduction of HOMA-IR during three months post-surgery in both groups of patients is depicted in Figure 1. 


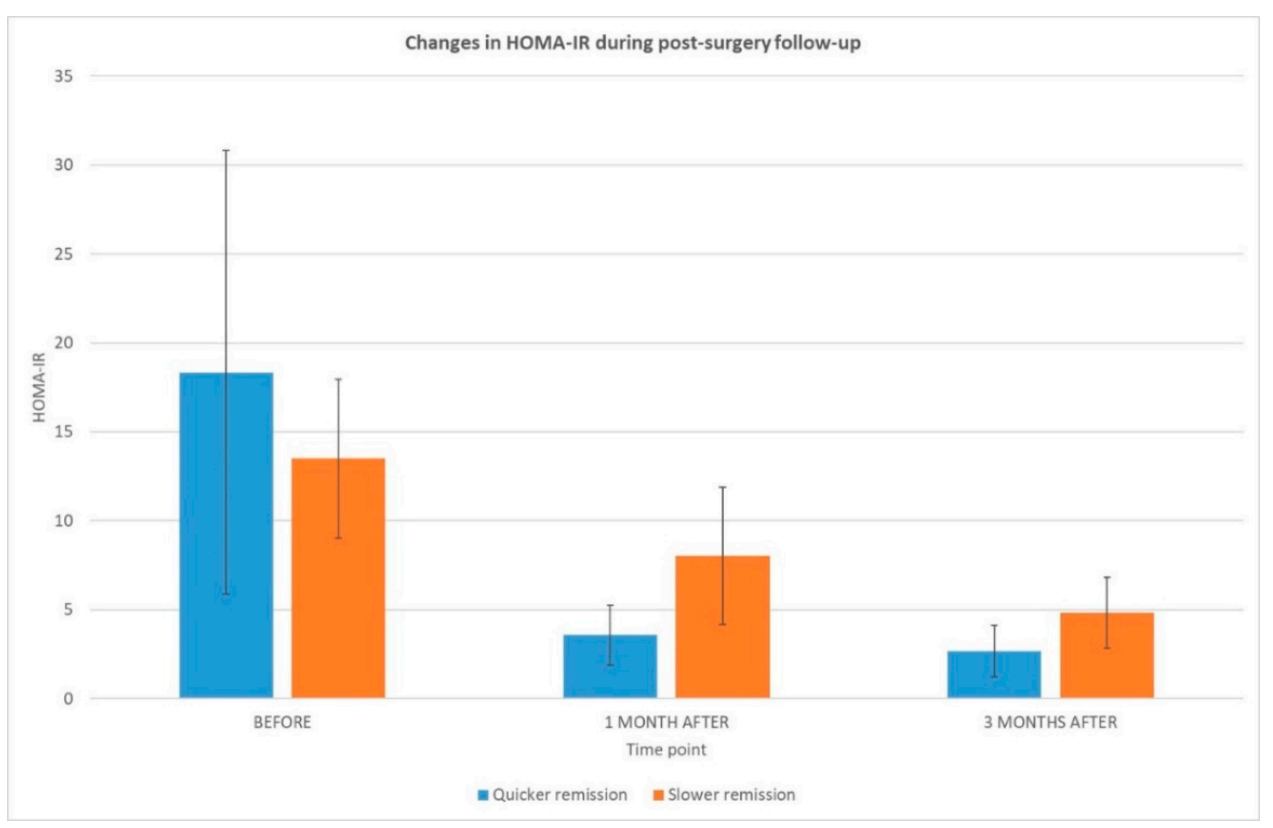

Figure 1. Changes in HOMA-IR (with its standard deviation) during follow-up after the bariatric surgical procedure in the groups with quicker (blue) and slower (orange) diabetes remission.

\subsection{Bioclinical Outcome}

At the beginning, the two established groups did not significantly differ in biochemical parameters. In both groups, laparoscopic sleeve gastrectomy evoked similar weight loss and similarly affected other parameters like glucose, cholesterol (including LDL and HDL) and TG concentrations. Due to the experiment's design between studied groups, significant differences in HOMA-IR reduction one and three months post-surgery were observed. Moreover, significantly different values for insulin and HOMA-IR were observed one month post-surgery. Consequently, we have focused on early (one month post-surgery) changes in plasma metabolome after bariatric interventions. Moreover, at this period of time, all patients had similar post-operative lifestyles which allowed us to get a possibly "clear" effect of surgery excluding, or with minimal impact from, other factors. Detailed patients' characteristics are presented in Table 1.

Table 1. Characteristics of study participants.

\begin{tabular}{|c|c|c|c|c|}
\hline $\begin{array}{l}\text { Time } \\
\text { Frame }\end{array}$ & Biochemical and Clinical Parameters & $\begin{array}{c}\text { Quicker } \\
\text { Remission }\end{array}$ & $\begin{array}{c}\text { Slower } \\
\text { Remission }\end{array}$ & $p$-Value \\
\hline & Age (years) & $47 \pm 10.3$ & $51 \pm 11.4$ & ns \\
\hline & Sex-F/M & $4 / 7$ & $4 / 5$ & - \\
\hline & Body Mass Index (BMI) before & $49 \pm 4.5$ & $51 \pm 8.3$ & ns \\
\hline & BMI 1 month after & $42 \pm 3.5$ & $46 \pm 8.1$ & ns \\
\hline & BMI 3 months after & $37 \pm 3.6$ & $43 \pm 7.6$ & ns \\
\hline & Excess weight loss (EWL, \%) 1 month after & $21 \pm 5.9$ & $18 \pm 4.8$ & ns \\
\hline & Excess weight loss (EWL, \%) 3 months after & $29 \pm 9.8$ & $29 \pm 7.4$ & ns \\
\hline & Weight loss after 1 month (kg) & $16 \pm 4.7$ & $15 \pm 2.8$ & ns \\
\hline & Time of type 2 diabetes (T2DM) lasting (months) & $30 \pm 59.3$ & $24 \pm 16.5$ & ns \\
\hline \multirow{8}{*}{  } & Glucose (mg/dL) & $158 \pm 62.6$ & $153 \pm 36.1$ & ns \\
\hline & Insulin $(\mathrm{pmol} / \mathrm{L})$ & $46 \pm 39.4$ & $36 \pm 13.8$ & ns \\
\hline & HOMA-IR & $18.3 \pm 12.5$ & $13.5 \pm 4.5$ & ns \\
\hline & Cholesterol (mg/dL) & $198 \pm 43.4$ & $186 \pm 27.7$ & ns \\
\hline & LDL (mg/dL) & $147 \pm 43.4$ & $114 \pm 29.2$ & ns \\
\hline & $\mathrm{HDL}(\mathrm{mg} / \mathrm{dL})$ & $50 \pm 22.8$ & $42 \pm 6.6$ & ns \\
\hline & $\mathrm{TG}(\mathrm{mg} / \mathrm{dL})$ & $150 \pm 55.2$ & $154 \pm 51.5$ & ns \\
\hline & A1c $(\%)$ & $6.88 \pm 1.3$ & $6.26 \pm 0.7$ & ns \\
\hline
\end{tabular}


Table 1. Cont.

\begin{tabular}{|c|c|c|c|c|}
\hline $\begin{array}{l}\text { Time } \\
\text { Frame }\end{array}$ & Biochemical and Clinical Parameters & $\begin{array}{l}\text { Quicker } \\
\text { Remission }\end{array}$ & $\begin{array}{c}\text { Slower } \\
\text { Remission }\end{array}$ & $p$-Value \\
\hline \multirow{9}{*}{ 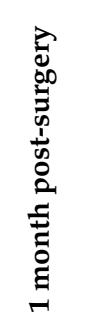 } & Glucose (mg/dL) & $102 \pm 20.3$ & $118 \pm 22.0$ & $\mathrm{~ns}$ \\
\hline & Insulin (pmol/L) & $13 \pm 7.7$ & $26 \pm 11.1$ & 0.011 \\
\hline & HOMA-IR & $3.6 \pm 1.7$ & $8.0 \pm 3.9$ & 0.029 \\
\hline & HOMA-IR decrease (\%) & $76 \pm 8.9$ & $42 \pm 16.2$ & 0.000 \\
\hline & Cholesterol (mg/dL) & $190 \pm 43.5$ & $197 \pm 28.7$ & ns \\
\hline & $\mathrm{LDL}(\mathrm{mg} / \mathrm{dL})$ & $126 \pm 38.0$ & $135 \pm 30.0$ & ns \\
\hline & $\mathrm{HDL}(\mathrm{mg} / \mathrm{dL})$ & $48 \pm 33.5$ & $59 \pm 43.9$ & ns \\
\hline & $\mathrm{TG}(\mathrm{mg} / \mathrm{dL})$ & $130 \pm 45.4$ & $149 \pm 75.5$ & ns \\
\hline & A1c $(\%)$ & $6.3 \pm 1.0$ & $5.9 \pm 0.4$ & ns \\
\hline \multirow{9}{*}{ 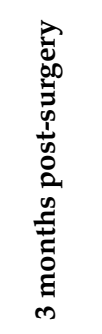 } & Glucose (mg/dL) & $99 \pm 17.7$ & $108 \pm 11.3$ & ns \\
\hline & Insulin (pmol/L) & $10 \pm 6.1$ & $18 \pm 8.5$ & ns \\
\hline & HOMA-IR & $2.67 \pm 1.5$ & $4.82 \pm 2.0$ & ns \\
\hline & HOMA-IR decrease (\%) & $80 \pm 12.3$ & $63 \pm 13.9$ & 0.011 \\
\hline & Cholesterol (mg/dL) & $184 \pm 46.8$ & $188 \pm 25.3$ & ns \\
\hline & $\mathrm{LDL}(\mathrm{mg} / \mathrm{dL})$ & $128 \pm 37.8$ & $132 \pm 35.4$ & $\mathrm{~ns}$ \\
\hline & $\mathrm{HDL}(\mathrm{mg} / \mathrm{dL})$ & $51 \pm 19.5$ & $47 \pm 11.8$ & ns \\
\hline & $\mathrm{TG}(\mathrm{mg} / \mathrm{dL})$ & $125 \pm 37.8$ & $139 \pm 28.7$ & ns \\
\hline & $\mathrm{A} 1 \mathrm{c}(\%)$ & $5.8 \pm 0.6$ & $6.5 \pm 1.3$ & ns \\
\hline
\end{tabular}

\subsection{Metabolomics}

Metabolomics analyses were performed with the previously described [7] LC-MS and GC-MS methods for serum fingerprinting. This resulted in the detection of over 50 statistically significant and identified metabolites.

The multivariate statistical analysis confirmed differences in the metabolome of examined groups. Before the surgery, all patients clustered together; however, their metabolic profiles differed after one-month post-surgery. Interestingly, the prediction of patients with slower remission by PLS-DA model built based on patients before and one-month post-surgery (Figure 2, left panel) placed the slower remission group exactly between the other groups (Figure 2, right panel).
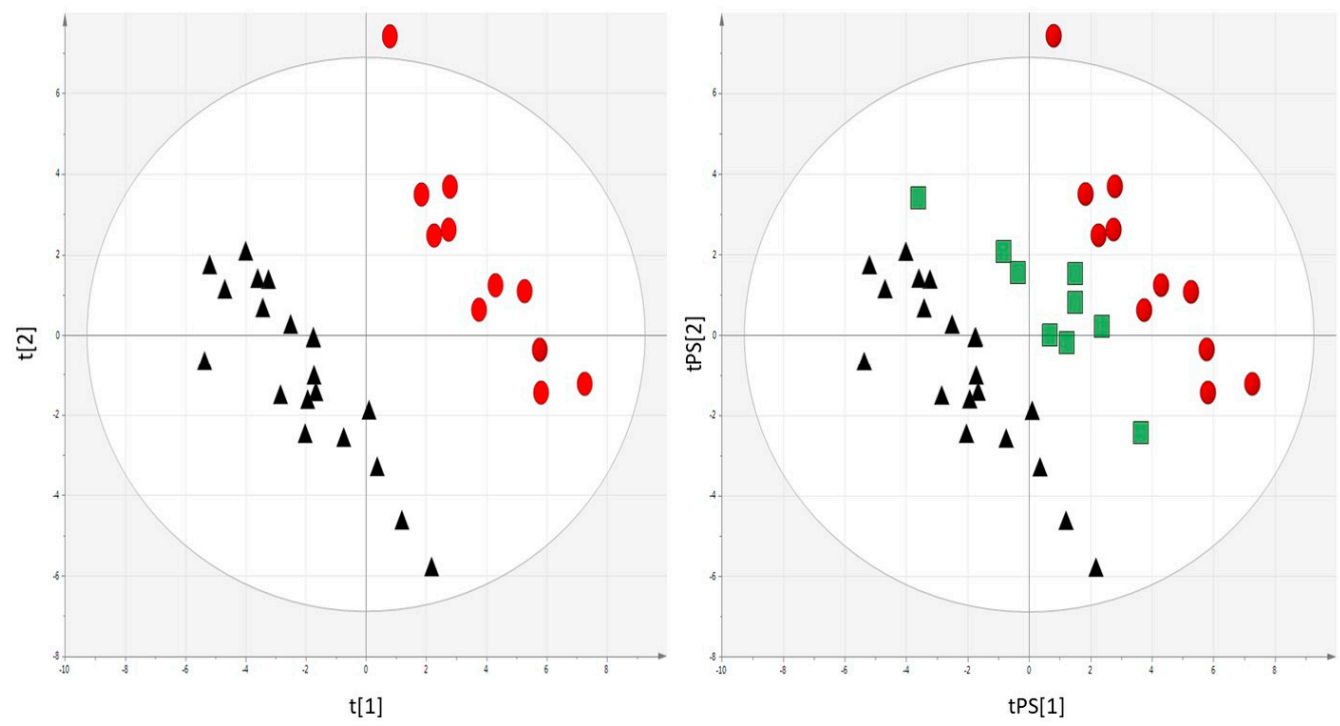

Figure 2. PLS-DA plots demonstrating the differences in the metabolome of the examined groups $(\boldsymbol{\Delta}$ - patients before the surgery, $\bullet$ - one month after and quicker remission, $\mathbf{\square}$-one month and slower remission). The left panel shows all patients clustered together before surgery and separated patients with quicker diabetes remission whose metabolic profiles differ after one-month post-surgery. A prediction of patients with slower remission by PLS-DA model built based on patients before and one-month post-surgery placed slower remission group exactly between the other groups (right panel). $R^{2}=0.947, Q^{2}=0.434 ;$ Pareto Scaling, Log transformed data. 
In the present study, only several compounds (detected by LC-MS, Table 2) were changed in a similar way in both groups. These metabolites are 2-ketoisocaproic acid, glycerol and the following classes of lipids: phosphatidylcholines (PC), lysophosphatidylcholines (LPC), phosphatidylethanolamines (PE), lysophosphatidylethanolamines (LPE) and fatty acids (FA).

Table 2. Metabolites altered similarly before and one month after the surgery in patients with quicker and slower type 2 diabetes (T2DM) improvement detected with liquid chromatography coupled with mass spectrometry (LC-MS).

\begin{tabular}{ccc}
\hline Metabolite & Quicker Remission & Slower Remission \\
\hline Lyso PC 14:0 (+) & $0.54^{* *}$ & $0.56^{*}$ \\
Lyso PC 20:5sn-2 (+) & $0.50^{* * * *}$ & $0.61^{*}$ \\
Lyso PC 20:3 (+) & $0.41^{* * * *}$ & $0.61^{*}$ \\
Lyso PC 20:5sn-1 (+) & $0.50^{* * * *}$ & $0.61^{*}$ \\
PC 30:0 (+) & $0.49^{* *}$ & $0.60^{*}$ \\
PC 32:1 (+) & $0.56^{* *}$ & $0.58^{* *}$ \\
PC 34:4 (+) & $0.48^{* *}$ & $0.54^{*}$ \\
PC 40:5(+) & $0.42^{* *}$ & $0.51^{*}$ \\
Lyso PE 18:1(-) & $0.72^{*}$ & $0.72^{*}$ \\
Lyso PE 20:5 (+/-) & $0.54^{* *}$ & $0.55^{*}$ \\
PE 16:0/20:5(-) & $0.52^{* *}$ & $0.56^{*}$ \\
arachidonic acid (-) & $1.34^{* *}$ & $1.31^{* *}$ \\
\hline
\end{tabular}

Alterations are presented as fold change (FC). PC: phosphatidylcholines; PE: phosphatidylethanolamines. $(+) /(-)$ - metabolite identified in positive or negative ESI mode, respectively; ns-respectively; ns-non-significant or significant but not identified; ${ }^{*} p \leq 0.05 ;{ }^{* *} p \leq 0.01 ;{ }^{* * *} p \leq 0.001 ;{ }^{* * * *} p \leq 0.0001$.

However, a much larger group of metabolites significantly changed one-month post-surgery in only one of the studied groups: quicker or slower T2DM remission (Table 3, 0 vs. 1 month columns). Again, in the case of LC-MS platform, significant metabolites can be classified mainly as PC, LPC, PE and LPE; additionally, changes in sphingomyelins (SM), fatty acids (FA) and fatty acid amides (FAAs) were observed. Some of them can be a component of biological membranes (PC, PE, SM); however, they can also be linked with type 2 diabetes (PC, PE, SM, FA), bacteria (PC, PE, SM) or oxidative stress (FAAs) $[7,13]$. The majority of them decreased post-surgery. This decrease was usually observed in the group with quicker diabetes remission. Additionally, some of them segregated in the studied groups before surgery or one-month after (Table 3, Quicker vs. Slower remission columns). It shows that a group with quicker remission already had lower levels of some lipids before the surgery or this level decreased quicker after the bariatric intervention. Only two metabolites increased after the surgery-PC 22:5/16:0 (FC $=1.27, p<0.05)$ and SM 33:2 (FC $=1.32, p<0.001)$ and this effect was observed only in the group with slower T2DM remission. Although the general tendency observed was a decrease of serum lipids after bariatric surgery, an increase of selected lipids has been already reported in other studies, including ours $[7,8]$.

Table 3. Metabolites discriminating quicker and slower T2DM remission groups detected with LC-MS.

\begin{tabular}{ccccc}
\hline \multirow{2}{*}{ Metabolite } & \multicolumn{2}{c}{ 0 vs. 1 Month } & \multicolumn{2}{c}{ Quicker vs. Slower Remission } \\
\cline { 2 - 5 } & $\begin{array}{c}\text { Quicker } \\
\text { Remission }\end{array}$ & $\begin{array}{c}\text { Slower } \\
\text { Remission }\end{array}$ & $\begin{array}{c}\text { Before } \\
\text { Surgery }\end{array}$ & $\begin{array}{c}\text { 1 Month } \\
\text { Post-Surgery }\end{array}$ \\
\hline L-Acetylcarnitine(+) & $2.24^{*}$ & $\mathrm{~ns}$ & $\mathrm{~ns}$ & $2.32^{*}$ \\
Linoleylcarnitine (+) & $1.45^{* *}$ & $\mathrm{~ns}$ & $\mathrm{~ns}$ & $\mathrm{~ns}$ \\
Elaidic carnitine (+) & $1.55^{* *}$ & $1.46^{*}$ & $\mathrm{~ns}$ & $\mathrm{~ns}$ \\
L-stearoylcarnitine (+) & $\mathrm{ns}$ & $0.60^{*}$ & $\mathrm{~ns}$ & $\mathrm{~ns}$ \\
Lactic acid (-) & $0.58^{*}$ & $\mathrm{~ns}$ & $\mathrm{~ns}$ & $\mathrm{~ns}$ \\
Uric acid (-) & $1.26^{*}$ & $\mathrm{~ns}$ & $\mathrm{~ns}$ & $\mathrm{~ns}$ \\
Oleic acid (-) & $1.40^{* *}$ & $1.39^{*}$ & $\mathrm{~ns}$ & $0.67^{*}$ \\
\hline
\end{tabular}


Table 3. Cont.

\begin{tabular}{|c|c|c|c|c|}
\hline \multirow[b]{2}{*}{ Metabolite } & \multicolumn{2}{|c|}{0 vs. 1 Month } & \multicolumn{2}{|c|}{ Quicker vs. Slower Remission } \\
\hline & $\begin{array}{c}\text { Quicker } \\
\text { Remission }\end{array}$ & $\begin{array}{c}\text { Slower } \\
\text { Remission }\end{array}$ & $\begin{array}{l}\text { Before } \\
\text { Surgery }\end{array}$ & $\begin{array}{c}1 \text { Month } \\
\text { Post-Surgery }\end{array}$ \\
\hline Oleamide $(+)$ & ns & $0.62^{* * * *}$ & ns & ns \\
\hline Stearamide $(+)$ & ns & $0.75 *$ & ns & ns \\
\hline Lyso PE 16:0 (-) & ns & ns & $0.56^{*}$ & ns \\
\hline Lyso PE P-16:0 (+) & ns & ns & $0.75 *$ & ns \\
\hline Lyso PE 18:0 (+/-) & $0.72 *$ & ns & $066^{*}$ & $0.65 *$ \\
\hline Lyso PE 18:1 (+/-) & ns & ns & $0.66^{*}$ & $0.63 *$ \\
\hline Lyso PE 18:2 (+/-) & $0.61^{* *}$ & ns & ns & $0.63 *$ \\
\hline Lyso PE 20:0 (-) & ns & ns & ns & $0.61 *$ \\
\hline Lyso PE 20:3 (+/-) & $0.51 *$ & ns & $0.63 *$ & $0.63 *$ \\
\hline Lyso PE 20:4 (-) & ns & ns & ns & $0.71 *$ \\
\hline Lyso PE 22:5 (-) & ns & ns & $0.55 *$ & $0.56 *$ \\
\hline PE 18:1/16:0 (-) & ns & $0.74 *$ & ns & ns \\
\hline PE 18:2/16:0 (-) & $0.61 *$ & ns & ns & ns \\
\hline PE 16:0/20:5 (-) & ns & ns & $0.70 *$ & ns \\
\hline PE 18:0/18:2 (-) & 0.50 * & ns & ns & ns \\
\hline PE 18:0/20:3 (-) & $0.54^{* * * *}$ & ns & ns & ns \\
\hline Lyso PC 14:0 (-) & 0.65 * & ns & ns & $0.57^{*}$ \\
\hline Lyso PC 15:0 (-) & ns & ns & $0.75 *$ & $0.69 *$ \\
\hline Lyso PC 16:0 (-) & ns & ns & $0.64 *$ & ns \\
\hline Lyso PC 16:1 (+) & $0.66^{*}$ & ns & ns & ns \\
\hline Lyso PC 17:1 (+) & ns & ns & $0.71 *$ & ns \\
\hline Lyso PC 18:0 (+/-) & $0.71^{* *}$ & ns & $0.72 *$ & $0.59 *$ \\
\hline Lyso PC 18:2(+/-) & $0.73 *$ & ns & ns & 0.69 * \\
\hline Lyso PC 20:1 (-) & ns & ns & $0.76^{*}$ & ns \\
\hline Lyso PC 20:2 (+/-) & $0.70^{* *}$ & ns & $0.70 *$ & ns \\
\hline Lyso PC 20:3 (+/-) & $0.60 *$ & ns & ns & ns \\
\hline Lyso PC 22:4 (+) & 0.70 * & ns & ns & ns \\
\hline Lyso PC 22:5 (+) & $0.61^{* *}$ & ns & $1.50 *$ & ns \\
\hline PC 30:0 (+) & ns & ns & ns & $0.64 *$ \\
\hline PC 32:2(+) & $0.49 * *$ & ns & ns & ns \\
\hline PC 34:3 (+) & $0.56^{* *}$ & ns & ns & ns \\
\hline PC 18:2/17:0 (-) & $0.68^{* *}$ & ns & ns & ns \\
\hline PC $38: 5(+)$ & $0.27 *$ & ns & ns & ns \\
\hline PC 22:5/16:0(-) & ns & $1.27 *$ & ns & ns \\
\hline SM 33:2(-) & ns & $1.32 * * *$ & ns & ns \\
\hline C14:1 sphingolipid (+) & $0.68^{* * * *}$ & ns & $0.49 *$ & ns \\
\hline Sphingosine $(+)$ & ns & ns & ns & $1.40 *$ \\
\hline Choline $(+)$ & ns & $0.70 *$ & $0.49 *$ & ns \\
\hline Lueucine/isoleucine (+) & ns & $0.62 * * * *$ & ns & ns \\
\hline Piperine (+) & ns & $0.58 *$ & $0.48 *$ & ns \\
\hline $\begin{array}{c}\text { Oxotetradecenoic acid or } \\
\text { hydroxytetradecenoic acid }(-)\end{array}$ & $2.06^{*}$ & ns & ns & ns \\
\hline Hydroperoxyoctadecadienoic acid (-) & $2.06 *$ & ns & ns & ns \\
\hline Succinyldiaminopimelic acid $(-)$ & ns & $0.75^{* * * *}$ & ns & ns \\
\hline Hydroxyandrostanone sulfate $(-)$ & $1.28^{* * * *}$ & $1.35 *$ & ns & ns \\
\hline Androsterone sulfate $(-)$ & $1.31^{* * * *}$ & ns & ns & ns \\
\hline Hydroperoxylinoleic acid (-) & $0.55 *$ & ns & ns & ns \\
\hline Taurine $(-)$ & ns & ns & $0.43 *$ & ns \\
\hline
\end{tabular}

Alterations are presented as fold change (FC). (+)/(-)-metabolite identified in positive or negative ESI mode, respectively; ns-non-significant or significant but not identified; ${ }^{*} p \leq 0.05 ; * * p \leq 0.01$; ${ }^{* * *} p \leq 0.001 ; * * * * p \leq 0.0001$. For lysophosphatidylcholines (LPCs) or lysophosphatidylethanolamines (LPEs), detected in both ion modes as well as for $s n-1$ and $s n-2$ isomers, an average FC is presented. 
In the case of GC-MS results: amino acids, organic acids, sugars, metabolites related to gut microbiota metabolism and others (Table 4 ) were found significantly segregating patients with quicker and slower T2DM remission.

Table 4. Metabolites segregating quicker and slower T2DM remission groups detected with a gas chromatography mass spectrometry (GC-MS).

\begin{tabular}{|c|c|c|c|c|}
\hline \multirow[b]{2}{*}{ Metabolite } & \multicolumn{2}{|c|}{0 vs. 1 Month } & \multicolumn{2}{|c|}{ Quicker vs. Slower Remission } \\
\hline & $\begin{array}{c}\text { Quicker } \\
\text { Remission }\end{array}$ & $\begin{array}{c}\text { Slower } \\
\text { Remission }\end{array}$ & $\begin{array}{l}\text { Before } \\
\text { Surgery }\end{array}$ & $\begin{array}{c}1 \text { Month } \\
\text { Post-Surgery }\end{array}$ \\
\hline 2-hydroxybutyric acid & $1.69 *$ & ns & ns & $1.70 *$ \\
\hline 3-hydroxybutyric acid & $7.32 * *$ & ns & ns & $4.76^{* *}$ \\
\hline 2-ketoisocaproic acid & $1.97 * *$ & $1.68 *$ & ns & ns \\
\hline phosphoric acid & $4.55 *$ & ns & ns & ns \\
\hline fumaric acid & $1.91 *$ & ns & ns & ns \\
\hline $\mathrm{L}-(+)$ lactic acid & ns & $\mathrm{ns}$ & $0.47^{*}$ & $\mathrm{~ns}$ \\
\hline citric acid & $2.75 *$ & ns & ns & $2.48^{* *}$ \\
\hline linoleic acid & ns & $\mathrm{ns}$ & ns & $1.61 *$ \\
\hline L-valine/norvaline & ns & 0.76 ** & ns & ns \\
\hline L-leucine & ns & $0.70 * *$ & ns & ns \\
\hline Isoleucine/norleucine & ns & $0.76^{*}$ & ns & $\mathrm{ns}$ \\
\hline L-threonine & ns & 0.73 * & ns & ns \\
\hline L-methionine & ns & $0.68 * *$ & ns & ns \\
\hline Phenylalanine & ns & $0.60 *$ & ns & ns \\
\hline L-tyrosine & ns & $0.62 * *$ & ns & ns \\
\hline L-tryptophan & ns & $0.63 * *$ & ns & ns \\
\hline L-alanine & ns & ns & 0.63 * & ns \\
\hline$N$-methylalanine & $1.81 *$ & ns & ns & ns \\
\hline Glycerol & $2.11^{* *}$ & 1.73 * & ns & $\mathrm{ns}$ \\
\hline ribose & ns & 0.44 * & ns & ns \\
\hline D-mannose/D-allose & ns & 0.69 * & ns & $\mathrm{ns}$ \\
\hline Furanose (tagatose) & $0.47^{* *}$ & ns & ns & ns \\
\hline $\begin{array}{l}\text { 2,3-Butanediol, } \\
\text { 2TMS derivative }\end{array}$ & ns & ns & $0.41^{* *}$ & ns \\
\hline
\end{tabular}

Alterations are presented as fold change (FC); ns-non-significant, ${ }^{*} p<0.05,{ }^{* *} p<0.01$.

\subsection{Correlation Analysis}

Spearman's rank correlation analysis of biochemical, clinical and metabolomics results was performed. Intensities of metabolites at 0 and one-month post-surgery were used for the correlation analysis. As a result, statistically significant correlations between carnitines, $\alpha-\mathrm{HB}, \beta-\mathrm{HB}$, citric acid, fatty acids, glycerol, taurine, amino acids (e.g., L-valine, L-isoleucine, L-leucine, L-tyrosine, L-threonine, L-methionine) and others with parameters like weight, BMI, HOMA-IR and its decrease, glucose and insulin concentrations, cholesterol (including LDL and HDL fractions), glycated haemoglobin and the $\%$ excess weight loss were observed. Selected results are listed below and depicted on Figure 2.

Carnitines correlated mainly with HOMA-IR decreased (L-acetylcarnitine: $\mathrm{r}_{\mathrm{S}}=0.738, p$-value $=$ 0.046; L-stearoylcarnitine: $\mathrm{r}_{\mathrm{S}}=0.761, p=0.037$; linoleylcarnitine: $\mathrm{r}_{\mathrm{S}}=-0.881, p=0.007$; elaidic carnitine: $\mathrm{r}_{\mathrm{S}}=-0.833, p=0.015$ ) in the group with quicker remission and with HOMA-IR (linoleylcarnitine and HOMA-IR before: $\mathrm{r}_{\mathrm{S}}=0.857, p=0.011$; elaidic carnitine and HOMA-IR before: $\mathrm{r}_{\mathrm{S}}=0.786, p=0.023$; linoleylcarnitine and HOMA-IR 1 month after: $\mathrm{r}_{\mathrm{S}}=-0.810, p=0.022$; elaidic carnitine and HOMA-IR 1 month after: $\mathrm{r}_{\mathrm{S}}=0.762, p=0.037$ ) and glucose level (linoleylcarnitine and glucose concentration one month after surgery: $\mathrm{r}_{\mathrm{S}}=0.826, p=0.017$; elaidic carnitine and glucose concentration after one month: $\left.\mathrm{r}_{\mathrm{S}}=0.778, p=0.030\right)$ in the group with slower T2DM remission. There was a correlation of $\alpha$-HB with cholesterol $\left(\mathrm{r}_{\mathrm{S}}=0.762, p=0.037\right)$, glucose $\left(\mathrm{r}_{\mathrm{S}}=0.838, p=0.013\right)$ and insulin concentration $\left(\mathrm{r}_{\mathrm{S}}=0.786, p=0.028\right)$, diabetes duration $\left(\mathrm{r}_{\mathrm{S}}=0.783, p=0.026\right)$, taurine $\left(\mathrm{r}_{\mathrm{S}}=0.905, p=0.005\right)$, citric acid $\left(\mathrm{r}_{\mathrm{S}}=0.738, p=0.046\right), 2$-ketoisocaproic acid $\left(\mathrm{r}_{\mathrm{S}}=-0.738, p=0.049\right)$ and L-valine $\left(\mathrm{r}_{\mathrm{S}}=0.738, p=0.037\right)$ 
in the group with slow remission and with weight $\left(\mathrm{r}_{S}=0.814, p=0.018\right)$, oleamide level $\left(\mathrm{r}_{\mathrm{S}}=-0.810\right.$, $p=0.022)$, 2-ketoisocaproic acid ( $\left.\mathrm{r}_{\mathrm{S}}=0.905, p=0.005\right)$ and L-valine $\left(\mathrm{r}_{\mathrm{S}}=-0.762, p=0.037\right)$ in the group with quicker remission. On the other hand, $\beta$-HB level correlated with glycated haemoglobin $\left(\mathrm{r}_{\mathrm{S}}=-0.778, p=0.030\right)$, oleic acid $\left(\mathrm{r}_{\mathrm{S}}=0.762, p=0.037\right)$ and uric acid $\left(\mathrm{r}_{\mathrm{S}}=0.786, p=0.028\right)$ in the group with rapid remission and with oleic acid $\left(\mathrm{r}_{\mathrm{S}}=0.738, p=0.046\right)$, L-stearoylcarnitine $\left(\mathrm{r}_{\mathrm{S}}=-0.857\right.$, $p=0.011)$, LDL concentration $\left(\mathrm{r}_{\mathrm{S}}=0.881, p=0.007 ; \mathrm{r}_{\mathrm{S}}=0.810, p=0.022\right)$, taurine $\left(\mathrm{r}_{\mathrm{S}}=0.833, p=0.015\right)$, L-stearoylcarnitine $\left(\mathrm{r}_{\mathrm{S}}=-0.857, p=0.011\right)$, 3-indolelactic acid $\left(\mathrm{r}_{\mathrm{S}}=0.857, p=0.011\right)$, pyruvic acid $\left(\mathrm{r}_{\mathrm{S}}=\right.$ $-0.857, p=0.011), \alpha-\mathrm{HB}\left(\mathrm{r}_{\mathrm{S}}=0.952, p=0.001\right), \mathrm{L}-\mathrm{valine}\left(\mathrm{r}_{\mathrm{S}}=0.738, p=0.046\right)$ and tagatose $\left(\mathrm{r}_{\mathrm{S}}=-0.833\right.$, $p=0.015)$ in the slower remission group. In the slower remission group, taurine correlated with BMI $\left(\mathrm{r}_{\mathrm{S}}=0.738, p=0.046\right)$, HDL $\left(\mathrm{r}_{\mathrm{S}}=-0.881, p=0.007\right), \mathrm{LDL}\left(\mathrm{r}_{\mathrm{S}}=0.857, p=0.011\right)$, HOMA-IR decrease $\left(\mathrm{r}_{\mathrm{S}}=0.738, p=0.046\right)$, weight $\left(\mathrm{r}_{\mathrm{S}}=0.929, p=0.002\right)$, L-valine $\left(\mathrm{r}_{\mathrm{S}}=0.762, p=0.037\right)$, L-isoleucine $\left(\mathrm{r}_{\mathrm{S}}\right.$ $=0.738, p=0.046)$, L-threonine $\left(\mathrm{r}_{\mathrm{S}}=0.905, p=0.005\right)$, phenylalanine $\left(\mathrm{r}_{\mathrm{S}}=0.833, p=0.015\right)$, fumaric acid ( $\left.\mathrm{r}_{\mathrm{S}}=0.857, p=0.011\right)$ and ribose $\left(\mathrm{r}_{\mathrm{S}}=0.762, p=0.037\right)$. In the rapid remission group, taurine correlated with HDL $\left(\mathrm{r}_{\mathrm{S}}=0.802, p=0.021\right)$, glucose $\left(\mathrm{r}_{\mathrm{S}}=0.833, p=0.015\right)$ and insulin $\left(\mathrm{r}_{\mathrm{S}}=0.762, p=\right.$ $0.037)$ concentration, HOMA-IR ( $\left.\mathrm{r}_{\mathrm{S}}=0.762, p=0.037\right)$, HOMA-IR decrease $\left(\mathrm{r}_{\mathrm{S}}=-0.762, p=0.037\right)$, weight loss ( $\left.\mathrm{r}_{\mathrm{S}}=-0.862, p=0.008\right)$, arachidonic acid $\left(\mathrm{r}_{\mathrm{S}}=0.857, p=0.011\right)$, stearic acid $\left(\mathrm{r}_{\mathrm{S}}=0.833\right.$, $p=0.015)$ and 3-indolelactic acid ( $\left.\mathrm{r}_{\mathrm{S}}=0.762, p=0.037\right)$.

A performed correlation analysis confirmed the importance of metabolites detected during metabolomics analysis, their connection with biochemical and clinical parameters, as well as observed differences between compared groups.

\subsection{Metabolic Pathways Analysis}

In the present study, MetaboAnalyst (Available online: http:/ /www.metaboanalyst.ca/), a general tool for metabolomics analysis [14], was used to link obtained metabolomics data with potential modulation of biochemical pathways. This platform is a comprehensive web-based tool suite designed to help users easily perform data analysis, visualization and functional interpretation [15].

Results from MetaboAnalyst showed that bariatric surgery induced changes in numerous metabolites that are involved in many biochemical pathways; however, arranged pathways are different or have different impact in both compared groups (Figure 3 and Table 5). In the group with quicker remission, pathways linked to an energetic process, such the citrate cycle (TCA cycle) ( $p$-value $=0.01$, impact 0.08$)$ or taurine metabolism $(p=0.01$, impact 0.36$)$, seems to be the most important. Synthesis and degradation of ketone bodies, which can be a link between energetic processes and diabetes, was also significantly altered $(p=0.047$, impact 0.00$)$. It is interesting to note the presence and importance of pathways related to microbiota-butanoate metabolism $(p=0.003$, impact $0.02)$, propanoate metabolism $(p=0.03$, impact 0.00$)$, and alanine, aspartate and glutamate metabolism $(p=0.014$, impact 0.06$)$. It can suggest mitochondria and microbiota activity alterations [16-18]. On the other hand, molecular alterations in the group with slower diabetes remission seem to be focused around amino acids metabolism. Important alterations in such pathways as BCAA biosynthesis $(p=0.00001$, impact 0.07$)$ and degradation $(p=0.001$, impact 0.06$)$, phenylalanine, tyrosine and tryptophan biosynthesis $(p=0.00418$, impact 0.01$)$, and glycine, serine and threonine metabolism $(p=0.02$, impact 0.10$)$ were found. Additionally, other pathways with lower impact, aminoacyl-tRNA biosynthesis $(p=0.0000001$, impact 0.00$)$, propanoate metabolism $(p=0.0087$, impact 0.00$)$ and nitrogen metabolism $(p=0.001$, impact 0.00$)$ were listed. 
L-Acetylcarnitine (before) L-Acetylcarnitine (after) Linoleylcarnitine (before) Linoleylcarnitine (after) Elaidic carnitine (before) Elaidic carnitine (after) L-stearoylcarnitine (before) L-stearoylcarnitine (after) 2-hydroxybutyric acid (before) 2-hydroxybutyric acid (after) 3-Hydroxybutyric acid (before) 3-Hydroxybutyric acid (after) Taurine (before)

Taurine (after)

Oleic acid (before)

Oleic acid (after)

Phosphoric acid (before) Phosphoric acid (after)
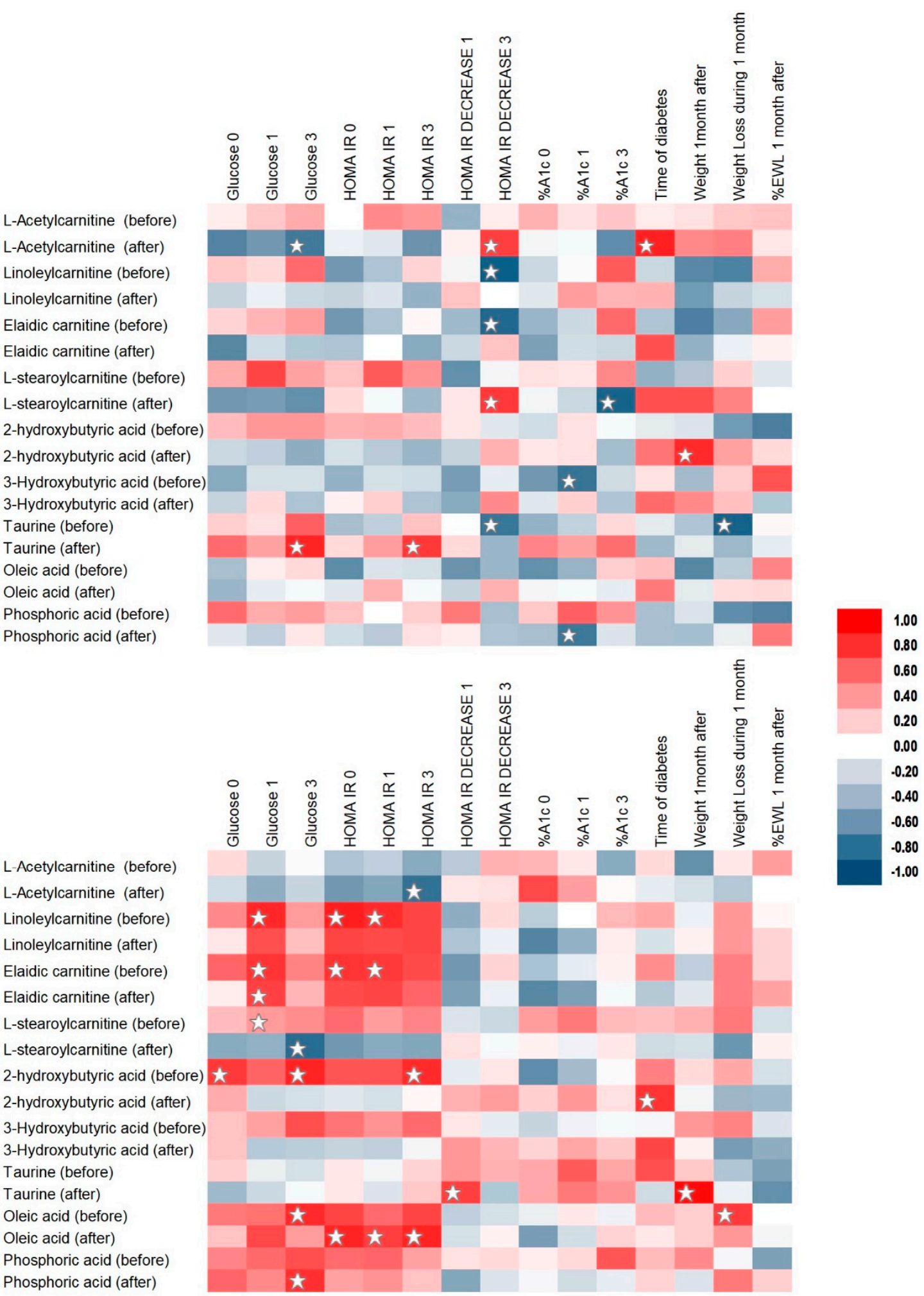

Figure 3. Selected Spearman correlation analysis results presented as heat maps for quicker (top) and slower (bottom) T2DM remission groups. Statistically significant correlations are marked with 放. Time points: 0 -before the surgery, 1 -one month after, 3-three months after; Metabolites: "before" - before the surgery, "after"—one month post-surgery. 
Table 5. Results (selected) from the Pathway Analysis.

\begin{tabular}{|c|c|c|c|c|}
\hline Group & Pathway & $p$-Value & FDR & Impact \\
\hline \multirow{7}{*}{$\begin{array}{l}\text { Quicker } \\
\text { remission }\end{array}$} & Butanoate metabolism & $3.43 \times 10^{-3}$ & $2.74 \times 10^{-1}$ & 0.02 \\
\hline & Citrate cycle & $1.03 \times 10^{-2}$ & $2.75 \times 10^{-1}$ & 0.08 \\
\hline & Taurine and hypotaurine metabolism & $1.03 \times 10^{-2}$ & $2.75 \times 10^{-1}$ & 0.36 \\
\hline & Alanine, aspartate and glutamate metabolism & $1.47 \times 10^{-2}$ & $2.94 \times 10^{-1}$ & 0.06 \\
\hline & Propanoate metabolism & $3.01 \times 10^{-2}$ & $4.81 \times 10^{-1}$ & 0.00 \\
\hline & Galactose metabolism & $4.03 \times 10^{-2}$ & $5.31 \times 10^{-1}$ & 0.03 \\
\hline & Synthesis and degradation of ketone bodies & $4.65 \times 10^{-2}$ & $5.31 \times 10^{-1}$ & 0.00 \\
\hline \multirow{8}{*}{$\begin{array}{l}\text { Slower } \\
\text { remission }\end{array}$} & Aminoacyl-tRNA biosynthesis & $1.44 \times 10^{-7}$ & $1.15 \times 10^{-5}$ & 0.00 \\
\hline & Valine, leucine and isoleucine biosynthesis & $1.42 \times 10^{-5}$ & $5.67 \times 10^{-4}$ & 0.07 \\
\hline & Nitrogen metabolism & $1.19 \times 10^{-3}$ & $2.63 \times 10^{-2}$ & 0.00 \\
\hline & Valine, leucine and isoleucine degradation & $1.31 \times 10^{-3}$ & $2.63 \times 10^{-2}$ & 0.06 \\
\hline & Phenylalanine, tyrosine and tryptophan biosynthesis & $4.18 \times 10^{-3}$ & $6.69 \times 10^{-2}$ & 0.01 \\
\hline & Propanoate metabolism & $8.74 \times 10^{-3}$ & $1.17 \times 10^{-1}$ & 0.00 \\
\hline & Glycine, serine and threonine metabolism & $2.07 \times 10^{-2}$ & $2.37 \times 10^{-1}$ & 0.10 \\
\hline & Taurine and hypotaurine metabolism & $2.48 \times 10^{-2}$ & $2.48 \times 10^{-1}$ & 0.36 \\
\hline
\end{tabular}

\section{Discussion}

In recent years, laparoscopic sleeve gastrectomy has become one of the most commonly used bariatric procedures, especially for morbid obesity. Although its role in diabetes treatment is still discussed; it is well documented that it leads to significant improvement in biochemical glucose homeostasis and can be a treatment method for patients with glucose metabolism abnormalities [10]. There are numerous hypotheses concerning potential causes of those effects, however, and many aspects of the metabolic effects of bariatric surgery remain unanswered $[2,11,19]$. In the meantime, an understanding of the metabolic changes induced by bariatric surgery may lead to new treatment strategies for obesity and related co-morbidities.

In our previous study, we presented the hypothesis that bariatric procedures in a semi-annual perspective lead to similar clinical effects, like weight loss or diabetes remission; however, molecular mechanisms involved in those outcomes varied dependently on bariatric procedure [7]. Instead, the study presented here shows that molecular mechanisms can also differ inside one surgery group. Here, these changes were demonstrated in the various pace of type 2 diabetes recovery. The performed metabolomics analyses suggest that mitochondria can play a crucial role in the process of bariatric surgery-induced T2DM recovery. However, the presence of microbe's related metabolites suggests significant alteration in gut microbiota activity and/or compositions. Additionally, an interesting finding was the detection of a group of metabolites which can be matched with changing mitochondria-microbes relation. This kind of connection could be, according to our knowledge, the first report on mitochondria influence and microbiota relationship to type 2 diabetes remission in the aspect of bariatric surgery. However, this effect should be confirmed by additional analyses. Performed correlation and pathways analyses have confirmed the crucial role of mitochondria as well as gut microbiota influence on T2DM recovery.

Mitochondrial metabolism is essential in maintaining physiological function in human cells, e.g., performing fatty acid oxidation and providing adenosine triphosphate (ATP) [19]. They are also a major producer of reactive oxygen species and reactive nitrogen species. Mitochondria are involved in numerous essential cell functions, e.g., energy metabolism, apoptotic pathways and steroid hormone synthesis. However, their function is reduced in insulin-responsive tissues in obesity and T2DM. Notably, insulin signaling was shown to impact mitochondrial DNA, protein synthesis and affect mitochondrial respiration and ATP production. No consensus has been reached on whether insulin resistance is a result of reduced mitochondrial density and whether it is the cause or consequence of mitochondrial dysfunction [19]. It was reported that destabilized in T2DM and obesity mitochondrial metabolism can be influenced by bariatric surgery. For example, lipid 
oxidation was higher in the diabetic group after bariatric surgery, which can suggest a different regulation of mitochondrial function in response to bariatric intervention in comparison to patients with normal glucose tolerance. Animal studies have shown a relation between Roux-en-Y gastric bypass and the mitochondrial fusion protein, Mfn1 levels, PGC1 $\alpha$ and NRF1 expression, citrate synthase activity and mitochondrial respiration, which has shown an association between RYGB and improved mitochondrial dynamics $[19,20]$. Detected significant alterations in lipids, carnitines, amino acids, $\alpha-\mathrm{HB}$ and $\beta-\mathrm{HB}$ and citric acid levels suggest meaningful modulation of mitochondria activity. Elevated levels of L-acetylcarnitine ( $\mathrm{FC}=2.24, p<0.05)$ and linoleylcarnitine $(\mathrm{FC}=1.45, p<0.01)$ were observed in the group with quicker T2DM remission, increased level of elaidic carnitine was observed in both groups ( $\mathrm{FC}=1.55, p$-value $<0.01$ in quicker and $\mathrm{FC}=1.46, p<0.05$ in slower remission group), while the level of L-stearoylcarnitine decreased in the slower remission group ( $\mathrm{FC}=0.6, p<0.05$ ). Carnitines are involved in the mitochondrial transport of fatty acid and are of critical importance for maintaining normal mitochondrial function. L-carnitine and its esters help to reduce oxidative stress, whereas acetyl-L-carnitine increases insulin sensitivity and glucose tolerance [21-23]. Fatty acid metabolism is dependent on mitochondria function; however, they may also be affected by impaired muscle BCAA catabolism. Plasma BCAAs are elevated in obese insulin resistant humans and their reduction was observed after bariatric surgery or weight loss [24]. Interestingly, during the first month of post-surgery, we observed a significant decline of amino acids only in the slower responders group. Nevertheless, there is a complex interplay between BCAA and lipids metabolism as well as the tricarboxylic acid (TCA) cycle, which leads to insulin resistance and T2DM development [25]. Considering the differences in the change of citric acid and fumaric acid, as well as $\alpha-\mathrm{HB}$ and $\beta-\mathrm{HB}$, the TCA cycle is also differentially affected in quicker and slower responders. Citric acid was found dramatically increased in the group with quick diabetes improvement $(\mathrm{FC}=2.75, p<0.05)$ and it was noticeably higher after the surgery in this group $(\mathrm{FC}=2.48, p<0.01)$. Citrate is an important substrate in cellular energy metabolism-it inhibits and induces important strategic enzymes located at the entrance and/or at the exit of glycolysis, TCA cycle, gluconeogenesis and fatty acids synthesis. Citric acid is synthesized in mitochondria from acetyl-CoA and oxaloacetate (OAA) and it becomes a substrate in the TCA cycle. Its subsequent complete oxidation provides the major source of cellular ATP production. Beyond the classical role as a metabolic regulator, its role in inflammation, cancer, insulin secretion, histone acetylation, disorders and diseases has been highlighted [26]. Fumaric acid is likewise a metabolite connected with the Krebs cycle. This dicarboxylic acid is a precursor to L-malate in the TCA cycle. In our study, its elevated level was observed in the group with rapid diabetes remission $(\mathrm{FC}=1.91, p<0.05)$. Uniquely, in the group with quick diabetes improvement, a noticeable increase in the level of $\alpha$-hydroxybutyric acid $(\mathrm{FC}=1.69, p<0.05)$ and $\beta$-hydroxybutyric acid (FC $=7.32, p<0.01)$ was observed. $\alpha$-HB is an organic acid derived from $\alpha$-ketobutyrate and is metabolized to propionyl-CoA and carbon dioxide. It was reported as an early marker for insulin resistance and impaired glucose regulation. The 2-hydroxybutyric acid level can elevate at least in two conditions-elevation of hepatic glutathione stress and elevation of the nicotinamide adenine dinucleotide (NADH)/nicotinamide adenine dinucleotide (NAD+) ratio due to increased lipid oxidation [13]. $\beta$-hydroxybutyric acid is the ketone body and energy carrier from adipocytes during fasting or exercise. Additionally, its downstream metabolism products have signaling activities. Metabolism of $\beta-\mathrm{HB}$ increases cellular levels of acetyl-CoA and reduces levels of succinyl-CoA and $\mathrm{NAD}+$. These can further increase mitochondrial protein acetylation and reduce mitochondrial protein succinylation, potentially regulating the function of many metabolic enzymes. The relative sparing of cytoplasmic NAD levels with a utilization of 3-hydroxybutyric acid rather than glucose can alter the activity of NAD-dependent enzymes such as sirtuins. Finally, $\beta-\mathrm{HB}$ might increase histone acetylation and alter gene expression by generativity acetyl-CoA in mitochondria, which can be transported into the nucleus via the citrate shuttle to serve as a substrate for histone acetyltransferases. These regulatory functions have important implications for the pathogenesis and treatment of metabolic diseases 
including type 2 diabetes [27]. Therefore, significant alterations of above mentioned metabolites suggest relation between the rate of T2DM recovery after bariatric surgery and energy metabolism.

Recent advances have also highlighted the impact of the gut microbiota on human health [18]. The microbiota offer many benefits to the host; however, gut dysbiosis (altered gut bacterial composition) was identified in overweight and obesity and marked as being associated with metabolic alterations such as insulin resistance, low-grade inflammation or adipocyte hypertrophy [28]. Data from human and animal studies have demonstrated that microbiota composition is modified after bariatric interventions. Bariatric surgery induces changes in the digestive tract anatomy and hormonal status; ingested nutrients and this modulation may impact the microbiota composition [29]. Detected alterations in the level of amino acids (especially BCAA), taurine, lactic acid, glycerol, $\alpha-\mathrm{HB}$ and $\beta-\mathrm{HB}$ and the contribution of butanoate metabolism, propanoate metabolism, alanine, aspartate and glutamate metabolism pathways (Figure 4, Table 5) suggest microbe's contribution in observed post-surgical molecular changes, including modulations related to T2DM remission.
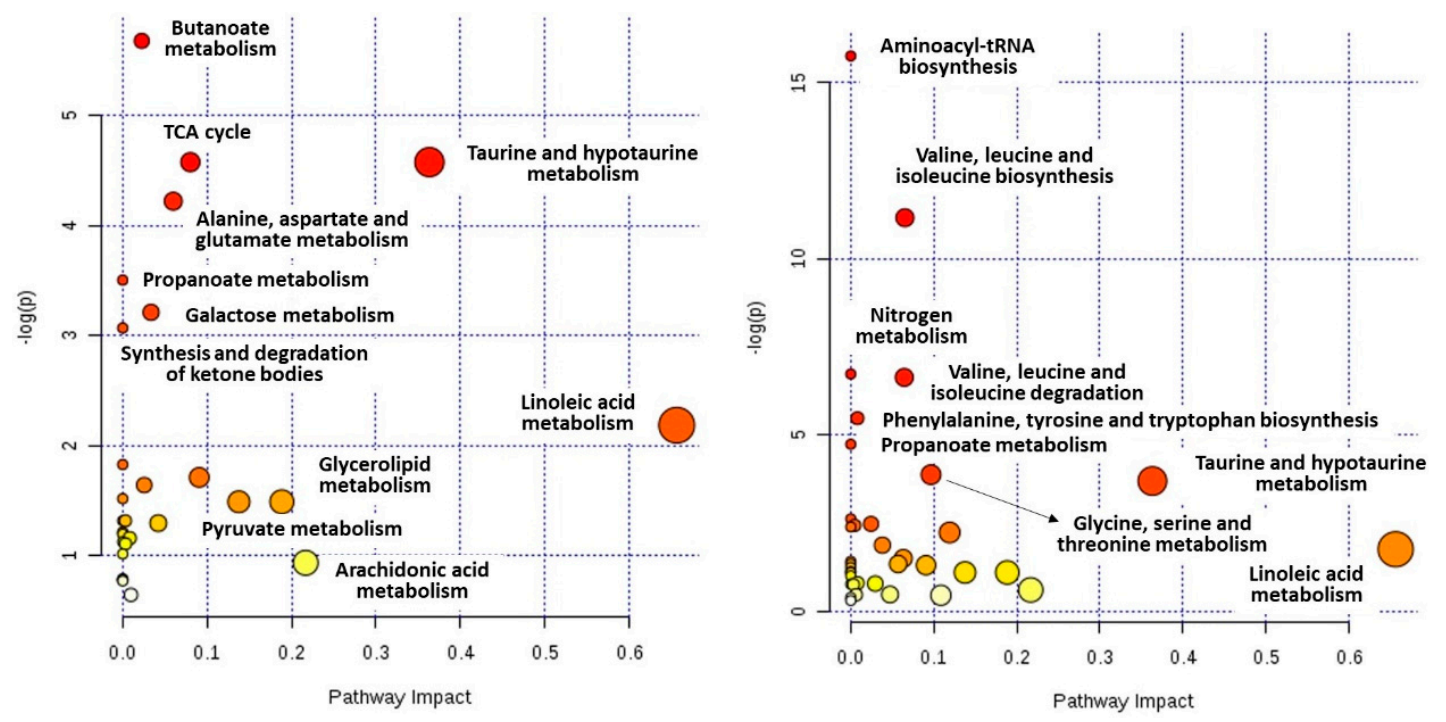

Figure 4. Summary of Pathway Analysis for group with quicker (left) and slower (right) diabetes remission.

In our study, the rapid remission group presented a base reduced taurine level in comparison to the slow remission group ( $\mathrm{FC}=0.43, p<0.05$ ). Taurine has a wide range of cytoprotective actions and inhibits the development of diet-related diseases such as diabetes and non-alcoholic fatty liver disease. The principal mechanism for its appearance in the intestine is secretion of taurine-conjugated bile acids followed by microbial deconjugation and the release of taurine. Its re-absorption is low, since taurine transporters are mostly located upstream in the small intestine; however, the Lactobacillus species are effective at deconjugating bile acids, specifically those conjugated to taurine. Additionally, bile acid deconjugation by Lactobacillus can be further stimulated by high glycolytic activity. Increased deconjugation of bile acids promotes the elimination of taurine by depleting endogenous taurine pools, which may reduce protection against complications of diabetes [30]. Among other microbe related metabolites, lactic acid can be mentioned. It decreased after one month-post surgery in the group with quicker remission ( $\mathrm{FC}=0.58, p<0.05$ ) and was lower in this group compared to before surgery $(\mathrm{FC}=0.47, p<0.05)$. Studies on animals have shown that specific strains of a lactic acid bacterium can be expected to be beneficial for the management of type 2 diabetes [31]. Lactate level reflects anaerobic metabolism and is a measurement of mitochondrial dysfunction from low mitochondrial oxidative capacity. Elevated lactate levels were observed in obese individuals and correlated positively with blood pressure and insulin resistance. Moreover, it was reported that lactate levels correlated inversely with the mitochondrial DNA copy number in PBMCs, suggesting the association between mitochondrial dysfunction and lactate levels [32]. The fermentation product 2,3-butanediol, which can 
be produced by fermenting bacteria, was reported as a factor which has a role in bacterial virulence, abundance and biofilm formation. It can also result in dynamic shifts in microbiota diversity [33]. In the presented study, a lower base level of the 2,3-butanediol derivative was observed in the group with rapid remission $(\mathrm{FC}=0.41, p<0.01)$. An interesting finding was an elevated level of phosphoric acid in the group with quicker diabetes remission ( $F C=4.55, p<0.05)$. It was reported that it showed antimicrobial activity and this action can be connected with increasing the hydrogen-ion concentration in the microorganism which cannot survive these conditions [34]. Microbe related metabolite is also an oxotetradecenoic acid, which was described as a component of bacterial lipopolysaccharide. Elevated levels ( $\mathrm{FC}=2.06, p<0.05$ ) were observed in the quicker remission group. Additionally, alterations in sulfate-containing metabolites, carnitines, amino acids (tryptophan, phenylalanine, tyrosine) or $\beta$-hydroxybutyric acid can be linked with gut microbes and their alterations [8].

Several studies have previously reported a bidirectional interaction between microbiota quality and mitochondrial function [16-18] and selected metabolites or pathways taking part in those interactions were observed in the described analyses. It is intriguing to note the presence of nitrogen related metabolites and the influence of nitrogen metabolism in the pathways analysis; however, its role can also be explained. The mitochondria-microbiota cross-talk is intriguing because mitochondria share many structural and functional features with the prokaryotic world. Moreover, this inter-talk may be crucial for human health—obesity, diabetes mellitus and Crohn's disease are associated with microbiota composition and different levels of Bacteroides and Firmicutes phyla $[16,18]$. Recent studies have highlighted the importance of a few key microbiome metabolites. The short-chain fatty acids (SCFA), especially butyrate and propionate, urolithins, lactic acid and lactate, selected carnitines, TCA related metabolites, lipopolysaccharides, flagelin, lipoteichoic acid, amino acids, fatty acids, dinitrophenol, hydrogen sulfide, nitric oxide, L-arginine and thiol can be mentioned here. $[17,18]$ Few reports have described how commensal or pathogenic microbiota can modulate mitochondrial function. In response to the microbe's activity, mitochondria can react in a few ways. Its main mechanisms are the regulation of energy production, alteration of redox balance, regulation of immune reactions by attenuating TNF $\alpha$-induced and inflammation-induced oxidation, which leads to mitochondrial dysfunction. On the other hand, mitochondria can modify microbiota composition through a production of reactive oxygen species and reactive oxygen nitrogen species, induction of the secretion of immune cells and enterochromaffin cells, modulation of gut functions (intestinal barrier function, mucosal immune response) mitochondrial genetic variants and heteroplasmy. An association between polymorphism of mitochondrial genes or D-Loop region in the mitochondrial genome and specific gut microbiota compositions was previously reported [16]. This can suggest that patients differ in gut microbiota compositions even before the surgery and that composition together with modulated mitochondrial activity can be an important factor in different T2DM remission tempos. The concentration of ROS directly correlated with the activity of the electron transfer chain, depending on its level, can induce differentiation or proliferation of a cell, cytokine release or apoptosis [18]. Additionally, increased ROS production may lead to lipid peroxidation. Interestingly, during the first month of post-surgery, we observed significant changes in oxidized fatty acids (Table 3), though only in the quicker remission group. The release of pathogenic lipopolysaccharydes, flagelin, lipoteichoic acid, lipoprotein or other toxins can generate signals which influenced specific receptors leading to an inflammatory response [18]. Some tend to increase ROS production by the mitochondrial respiratory chain. Activation of the immune system generally increases ATP production in lymphocytes, usually due to high glycolytic activity and mitochondrial fatty acid oxidation related with a reduction of electron transfer chain gene expression. Related to the Warburg effect, high glycolytic activity leads to a high production of different precursors involved in the biosynthesis of nicotinamide adenine dinucleotide phosphate (NADPH), amino acids, nucleotides and fatty acids. Additionally, stimulation of lymphocyte B causes upregulation of glycolysis and oxidative phosphorylation [18]. Dinitrophenol increases ROS production and affects epithelial barrier function leading to structurally abnormal mitochondria $[17,18]$. Due to the degradation of sulfur amino acids in the gut, some bacteria species 
can produce a large amount of hydrogen sulfide. Its elevated concentration inhibits cytochrome oxidase - one of the major complexes of the mitochondrial respiratory chain. Nitric oxide is produced by the host during inflammation due to L-arginine conversion or nitrate reduction, which is suppressed energy metabolism by reducing acetyl-CoA production. Thus, the presence of microbiota-related factors may affect mitochondria activity and ROS production [18]. The aforementioned butyrate is known to be produced by bacteria. $[8,17,35]$ It can enter the citric acid cycle to reduce $\mathrm{NAD}^{+}$to $\mathrm{NADH}$ and can be used as a carbon source even in the presence of glucose. Thus, butyrate regulates mitochondrial activity and promotes the release of signaling hormones like GLP-1 related to lower food intake [18].

The obtained results indicate a relationship between gut microbiota and mitochondria modulation by bariatric surgery and consequent type 2 diabetes remission. All data and relationships presented above allowed us to conclude that a key player in diabetes remission can be mitochondria and their capability. However, gut microbiota can also be a determinative factor that can regulate their function. Observed findings are novel, and further research using specific microbiology and mitochondria-related measurements are needed for their confirmation and to give a full explanation of the exact molecular mechanisms.

\section{Materials and Methods}

\subsection{Materials}

\subsubsection{Study Participants}

The study participants were selected from a cohort of 372 obese individuals who underwent laparoscopic sleeve gastrectomy in the 1st Clinical Department of General and Endocrine Surgery (Medical University of Bialystok, Bialystok, Poland). From this cohort, 20 patients with T2DM, but without post-surgery pharmacological anti-diabetes treatment, were selected. A majority of the experimental subjects had newly diagnosed T2DM and they did not receive anti-diabetic treatment before and after the surgery. Only a few patients from both groups were taking metformin before the surgery; however, the treatment was discontinued immediately after the performed surgery. Type 2 diabetes was recognized according to the American Diabetes Association's criteria when fasting plasma glucose was $\geq 126 \mathrm{mg} / \mathrm{dL}$, or $2 \mathrm{~h}$ plasma glucose in the $2 \mathrm{~h}-75 \mathrm{~g}$ OGTT $\geq 200 \mathrm{mg} / \mathrm{dL}$, or HbA1c $\geq 6.5 \%$. Based on biochemical and clinical parameters, with special impact to HOMA-IR reduction, patients were divided into two groups with a quicker or slower rate of type 2 diabetes remission. Both groups were matched in age, sex, BMI, diet and other basic parameters. Detailed characteristics of the study groups are presented in Table 1. Metabolomics analyses were performed on fasting serum samples obtained before and one month after surgery. The study was performed with the approval of the Ethics Committee at the Medical University of Bialystok (R-I-002/531/2013, 28 November 2013), upon written consent from all participants.

\subsubsection{Samples Collection}

Venous blood was drawn from participants in the fasting state into syringes containing a clotting activator (serum) or $\mathrm{NaF} / \mathrm{Na}_{2}$ EDTA (plasma). To obtain the serum, the whole blood was allowed to clot by leaving it undisturbed at room temperature for $30 \mathrm{~min}$. Serum and plasma were obtained by centrifugation at $1300 \times \mathrm{g}$ for $30 \mathrm{~min}$ in $4{ }^{\circ} \mathrm{C}$. Aliquots of the samples were stored at $-80{ }^{\circ} \mathrm{C}$ until analysis.

\subsubsection{Chemicals}

Purified water was obtained using the Milli-Qplus185 system (Millipore, Billerica, MA, USA). LC-MS grade methanol $(\mathrm{MeOH})$, acetonitrile $(\mathrm{ACN})$, formic acid and LC grade ethanol (EtOH), standard mix for GC-MS, containing grain fatty acid methyl ester mixture (C8-C28), 2-propanol and 
analytical grade heptane were purchased from Sigma-Aldrich ChemieGmbH (Steinheim, Germany). C18:0methyl ester and $\mathrm{N}, \mathrm{O}$-bis(trimethylsilyl) trifluoroacetamide with $1 \%$ trimethylchlorosilane were obtained from Pierce Chemical Co (Rockford, IL, USA). Silylation grade pyridine was purchased from VWR International BHDProlabo (Madrid, Spain). The API-TOF reference mass solution kit (G1969-850001) and tuning solutions, ESI-L low concentration tuning mix (G1969-85000) and ESI-TOF Biopolymer Analysis referencemasses (G1969-850003) were purchased from Agilent Technologies (Santa Clara, CA, USA).

\subsection{Methods}

\subsubsection{Biochemical Measurements}

Plasma glucose concentrations were analyzed by a hexokinase method (Roche Diagnostics International Ltd., Rotkreuz, Switzerland) and plasma level of HbA1c was measured by the HPLC method (Bio-Rad VARIANT). During these measurements, external and internal quality controls were positioned within the allowed ranges.

The HOMA-IR index was estimated using the following formula:

$$
\text { HOMA-IR = fasting insulin }(\mathrm{mIU} / \mathrm{L}) \times \text { fasting glucose }(\mathrm{mmol} / \mathrm{L}) / 22.5
$$

\subsubsection{Metabolic fingerprinting by LC-MS and GC-MS}

Metabolic fingerprinting was accomplished on LC-MS (6550, Agilent Technologies, Santa Clara, CA, USA) and GC-MS (5975C, Agilent Technologies, Santa Clara, CA, USA) platforms. Serum samples preparation, analysis and data treatment were performed according to the previously described protocols [7].

The Identification of metabolites detected by LC-MS was performed only for statistically significant metabolic features. The identity of compounds was confirmed by LC-MS/MS using a QTOF (model 6550, Agilent). Obtained MS/MS spectra were compared with the spectral data of reference compounds (HMDB, METLIN, LIPIDMAPS through CEU mass mediator), and product ions of commercially (Sigma) available reagents with those obtained in real samples. Phospholipids, lysophospholipids and sphingomyelins were confirmed with recently described characteristic fragments [36].

\subsubsection{Statistical Analysis}

Statistical analysis was performed to identify the differences in serum metabolites between two groups of patients established based on the information about T2DM remission rate. Primarily, serum samples obtained before surgery were compared in order to determine whether prior surgery metabolic profiles between patients with quicker and slower T2DM remissions were different. The other comparison was performed to find exact metabolic changes during the month after the surgery in patients with faster and slower remissions of T2DM. For the comparison of patients before surgery, a selection of statistically significant metabolites was performed using a $t$-test or Mann-Whitney U-test (depending on the normality of data distribution). To compare patients in time (before and after the surgery), depending on the data distribution, a paired $t$-test or Wilcoxon signed rank test was used. The normality of data distribution was assessed using the Shapiro-Wilk test. Equality of variance for compared groups was tested by an F-test. The $t$-test, paired $t$-test and F-test were calculated with Excel (Microsoft Corporation, Redmond, WA, USA) while the U-test, Wilcoxon signed rank test, Shapiro-Wilk tests and Spearman's rank correlation coefficient were performed with MATLAB 7.10 R2010a (MathWorks Inc., Natick, MA, USA). 


\section{Conclusions}

In this study, we presented that a modified level of carnitines, lipids, amino acids and $\alpha$ - and $\beta$-hydroxybutyric acids suggest a major role of mitochondria activity in the T2DM remission process. Additionally, some of the observed metabolites suggested that post-surgery modulation of gut microbiota composition may correlate with diabetes' recovery tempo. Additional analyses confirmed a relationship between biochemical and clinical parameters and aforementioned metabolites, thereby highlighting the role of mitochondria and microbes in the rate of T2DM remission.

According to our knowledge, the correlation between bariatric surgery evoked mitochondria and gut microbiota modulation and type 2 diabetes remission has been presented here for the first time. More investigation is needed to confirm and explore these findings. Therefore, we believe that the presented results indicate novel directions of research. Examination of mitochondria modulation and gut microbiota activity after bariatric surgery may help to reveal the mechanism by which this type of surgery leads to T2DM remission.

Author Contributions: Conceptualization: P.S., H.R.H., J.D., C.B., A.K., M.C.; Methodology: P.S., E.A.-P., A.C., M.C.; Software: P.S., M.C.; Investigation: P.S., H.R.H., E.A.-P., A.C., J.D.; Data Curation: P.S., E.A.-P., A.C., M.C.; Writing-Original Draft Preparation: P.S., M.C.; Writing-Review \& Editing: P.S., C.B., A.K., M.C.; Visualization: P.S., M.C.; Supervision: J.D., C.B., A.K., M.C.; Final approval of the version to be submitted: P.S., H.R.H., E.A.-P., A.C., J.D., C.B., A.K., M.C.

Funding: Project was supported by funds from the Leading National Research Center in Bialystok (108/KNOW/15 and 30/KNOW/2013).

Acknowledgments: The authors would like to thank Dan Cherry for his careful English editing and proofreading.

Conflicts of Interest: The authors declare no conflict of interest.

\section{Abbreviations}

$\begin{array}{ll}\alpha \text {-HB } & \alpha \text {-hydroxybutyric acid/2-hydroxybutyric acid } \\ \beta \text {-HB } & \beta \text {-hydroxybutyric acid/3-hydroxybutyric acid } \\ \text { acetyl-CoA } & \text { acetyl coenzyme A } \\ \text { ATP } & \text { Adenosine triphosphate } \\ \text { BCAAs } & \text { branched-chain amino acids } \\ \text { BMI } & \text { body mass index } \\ \text { CLR } & \text { C-type lectin receptor } \\ \text { (\%)EWL } & \text { (\%) excess weight loss } \\ \text { FA } & \text { fatty acid } \\ \text { FAA } & \text { fatty acid amides } \\ \text { FC } & \text { fold change } \\ \text { FDR } & \text { false discovery rate procedure } \\ \text { GC } & \text { gas chromatography } \\ \text { HbA1c } & \text { hemoglobin A1c (glycated hemoglobin) } \\ \text { HDL } & \text { high-density lipoprotein } \\ \text { HOMA } & \text { homeostatic model assessment } \\ \text { IR } & \text { insulin resistance } \\ \text { LC } & \text { liquid chromatography } \\ \text { LDL } & \text { low-density lipoprotein } \\ \text { (L)PE } & \text { (lyso)phosphatidylethanolamine } \\ \text { (L)PC } & \text { (lyso)phosphatidylcholine } \\ \text { LPS } & \text { lipopolysaccharide } \\ \text { LSG } & \text { laparoscopic sleeve gastrectomy } \\ \text { Mfn1 } & \text { Mitofusin 1 } \\ \text { MS } & \text { mass spectrometry } \\ \text { NAD } & \text { nicotinamide adenine dinucleotide } \\ \text { NADH } & \text { nicotinamide adenine dinucleotide } \\ & \end{array}$




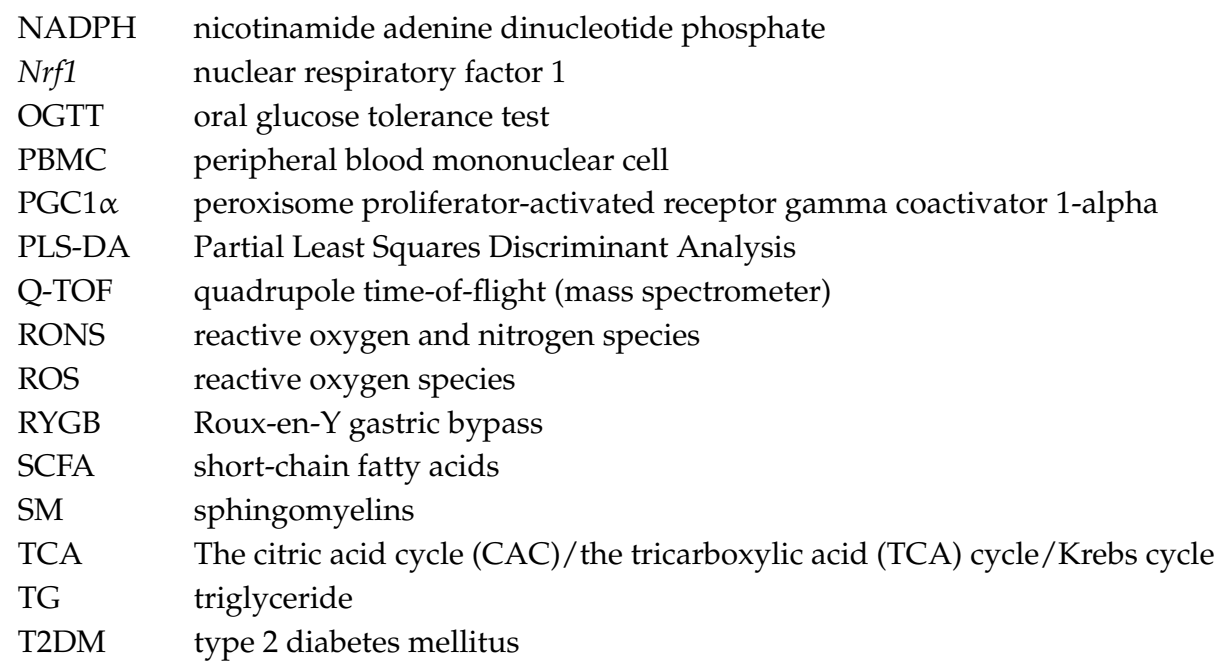

\section{References}

1. Kamvissi-Lorenz, V.; Raffaelli, M.; Bornstein, S.; Mingrone, G. Role of the Gut on Glucose Homeostasis: Lesson Learned from Metabolic Surgery. Curr. Atheroscler. Rep. 2017, 19, 9. [CrossRef] [PubMed]

2. Lee, W.-J.; Almalki, O. Mechanism of diabetes control after metabolic surgery. Ann. Laparosc. Endosc. Surg. 2017, 2, 128. [CrossRef]

3. Nguyen, N.T.; Varela, J.E. Bariatric surgery for obesity and metabolic disorders: State of the art. Nat. Rev. Gastroenterol. Hepatol. 2017, 14, 160-169. [CrossRef] [PubMed]

4. Celiker, H. A new proposed mechanism of action for gastric bypass surgery: Air hypothesis. Med. Hypotheses 2017, 107, 81-89. [CrossRef] [PubMed]

5. Goh, Y.M.; Toumi, Z.; Date, R.S. Surgical cure for type 2 diabetes by foregut or hindgut operations: A myth or reality? A systematic review. Surg. Endosc. 2017, 31, 25-37. [CrossRef] [PubMed]

6. Kassem, M.A.M.; Durda, M.A.; Stoicea, N.; Cavus, O.; Sahin, L.; Rogers, B. The Impact of Bariatric Surgery on Type 2 Diabetes Mellitus and the Management of Hypoglycemic Events. Front. Endocrinol. (Lausanne) 2017, 8, 37. [CrossRef] [PubMed]

7. Samczuk, P.; Luba, M.; Godzien, J.; Mastrangelo, A.; Hady, H.R.; Dadan, J.; Barbas, C.; Gorska, M.; Kretowski, A.; Ciborowski, M. "Gear mechanism" of bariatric interventions revealed by untargeted metabolomics. J. Pharm. Biomed. Anal. 2018, 151, 219-226. [CrossRef] [PubMed]

8. Samczuk, P.; Ciborowski, M.; Kretowski, A. Application of Metabolomics to Study Effects of Bariatric Surgery. J. Diabetes Res. 2018, 2018, 6270875. [CrossRef] [PubMed]

9. Lee, W.J.; Almulaifi, A.; Tsou, J.J.; Ser, K.H.; Lee, Y.C.; Chen, S.C. Laparoscopic sleeve gastrectomy for type 2 diabetes mellitus: Predicting the success by ABCD score. Surg. Obes. Relat. Dis. 2015, 11, 991-996. [CrossRef] [PubMed]

10. Major, P.; Wysocki, M.; Pędziwiatr, M.; Małczak, P.; Pisarska, M.; Budzyński, A. Laparoscopic sleeve gastrectomy for the treatment of diabetes mellitus type 2 patients-Single center early experience. Gland Surg. 2016, 5, 465-472. [CrossRef] [PubMed]

11. Koliaki, C.; Liatis, S.; le Roux, C.W.; Kokkinos, A. The role of bariatric surgery to treat diabetes: Current challenges and perspectives. BMC Endocr. Disord. 2017, 17, 50. [CrossRef] [PubMed]

12. Benaiges, D.; Flores-Le Roux, J.A.; Pedro-Botet, J.; Chillaron, J.J.; Renard, M.; Parri, A.; Ramon, J.M.; Pera, M.; Goday, A. Sleeve gastrectomy and Roux-en-Y gastric bypass are equally effective in correcting insulin resistance. Int. J. Surg. 2013, 11, 309-313. [CrossRef] [PubMed]

13. Gall, W.E.; Beebe, K.; Lawton, K.A.; Adam, K.-P.; Mitchell, M.W.; Nakhle, P.J.; Ryals, J.A.; Milburn, M.V.; Nannipieri, M.; Camastra, S.; et al. $\alpha$-Hydroxybutyrate Is an Early Biomarker of Insulin Resistance and Glucose Intolerance in a Nondiabetic Population. PLoS ONE 2010, 5, e10883. [CrossRef] [PubMed]

14. Cuperlovic-Culf, M. Machine Learning Methods for Analysis of Metabolic Data and Metabolic Pathway Modeling. Metabolites 2018, 8, 4. [CrossRef] [PubMed] 
15. Chong, J.; Soufan, O.; Li, C.; Caraus, I.; Li, S.; Bourque, G.; Wishart, D.S.; Xia, J. MetaboAnalyst 4.0: Towards more transparent and integrative metabolomics analysis. Nucleic Acids Res. 2018, 46, W486-W494. [CrossRef] [PubMed]

16. Clark, A.; Mach, N. The Crosstalk between the Gut Microbiota and Mitochondria during Exercise. Front. Physiol. 2017, 8, 319. [CrossRef] [PubMed]

17. Franco-Obregon, A.; Gilbert, J.A. The Microbiome-Mitochondrion Connection: Common Ancestries, Common Mechanisms, Common Goals. mSystems 2017, 2. [CrossRef] [PubMed]

18. Saint-Georges-Chaumet, Y.; Edeas, M. Microbiota-mitochondria inter-talk: Consequence for microbiota-host interaction. Pathog. Dis. 2016, 74, ftv096. [CrossRef] [PubMed]

19. Dankel, S.N.; Staalesen, V.; Bjorndal, B.; Berge, R.K.; Mellgren, G.; Burri, L. Tissue-specific effects of bariatric surgery including mitochondrial function. J. Obes. 2011, 2011, 435245. [CrossRef] [PubMed]

20. Sacks, J.; Mulya, A.; Fealy, C.E.; Huang, H.; Mosinski, J.D.; Pagadala, M.R.; Shimizu, H.; Batayyah, E.; Schauer, P.R.; Brethauer, S.A.; et al. Effect of Roux-en-Y gastric bypass on liver mitochondrial dynamics in a rat model of obesity. Physiol. Rep. 2018, 6. [CrossRef] [PubMed]

21. Sharma, S.; Black, S.M. Carnitine homeostasis, mitochondrial function, and cardiovascular disease. Drug Discov. Today Dis. Mech. 2009, 6, e31-e39. [CrossRef] [PubMed]

22. Pekala, J.; Patkowska-Sokola, B.; Bodkowski, R.; Jamroz, D.; Nowakowski, P.; Lochynski, S.; Librowski, T. L-carnitine-Metabolic functions and meaning in humans life. Curr. Drug Metab. 2011, 12, 667-678. [CrossRef] [PubMed]

23. Ruggenenti, P.; Cattaneo, D.; Loriga, G.; Ledda, F.; Motterlini, N.; Gherardi, G.; Orisio, S.; Remuzzi, G. Ameliorating hypertension and insulin resistance in subjects at increased cardiovascular risk: Effects of acetyl-L-carnitine therapy. Hypertension 2009, 54, 567-574. [CrossRef] [PubMed]

24. Lerin, C.; Goldfine, A.B.; Boes, T.; Liu, M.; Kasif, S.; Dreyfuss, J.M.; De Sousa-Coelho, A.L.; Daher, G.; Manoli, I.; Sysol, J.R.; et al. Defects in muscle branched-chain amino acid oxidation contribute to impaired lipid metabolism. Mol. Metab. 2016, 5, 926-936. [CrossRef] [PubMed]

25. Newgard, C.B. Interplay between lipids and branched-chain amino acids in development of insulin resistance. Cell Metab. 2012, 15, 606-614. [CrossRef] [PubMed]

26. Iacobazzi, V.; Infantino, V. Citrate-New functions for an old metabolite. Biol. Chem. 2014, 395, 387-399. [CrossRef] [PubMed]

27. Newman, J.C.; Verdin, E. beta-hydroxybutyrate: Much more than a metabolite. Diabetes Res. Clin. Pract. 2014, 106, 173-181. [CrossRef] [PubMed]

28. Aron-Wisnewsky, J.; Prifti, E.; Belda, E.; Ichou, F.; Kayser, B.D.; Dao, M.C.; Verger, E.O.; Hedjazi, L.; Bouillot, J.L.; Chevallier, J.M.; et al. Major microbiota dysbiosis in severe obesity: Fate after bariatric surgery. Gut 2018. [CrossRef] [PubMed]

29. Aron-Wisnewsky, J.; Dore, J.; Clement, K. The importance of the gut microbiota after bariatric surgery. Nat. Rev. Gastroenterol. Hepatol. 2012, 9, 590-598. [CrossRef] [PubMed]

30. Silva, J.C.P.; Mota, M.; Martins, F.O.; Nogueira, C.; Goncalves, T.; Carneiro, T.; Pinto, J.; Duarte, D.; Barros, A.S.; Jones, J.G.; et al. Intestinal Microbial and Metabolic Profiling of Mice Fed with High-Glucose and High-Fructose Diets. J. Proteome Res. 2018, 17, 2880-2891. [CrossRef] [PubMed]

31. Honda, K.; Moto, M.; Uchida, N.; He, F.; Hashizume, N. Anti-diabetic effects of lactic acid bacteria in normal and type 2 diabetic mice. J. Clin. Biochem. Nutr. 2012, 51, 96-101. [CrossRef] [PubMed]

32. Gamboa, J.L.; Billings, F.T.; Bojanowski, M.T.; Gilliam, L.A.; Yu, C.; Roshanravan, B.; Roberts, L.J.; Himmelfarb, J.; Ikizler, T.A.; Brown, N.J. Mitochondrial dysfunction and oxidative stress in patients with chronic kidney disease. Physiol. Rep. 2016, 4. [CrossRef] [PubMed]

33. Nguyen, M.; Sharma, A.; Wu, W.; Gomi, R.; Sung, B.; Hospodsky, D.; Angenent, L.T.; Worgall, S. The fermentation product 2,3-butanediol alters P. aeruginosa clearance, cytokine response and the lung microbiome. ISME J. 2016, 10, 2978-2983. [CrossRef] [PubMed]

34. Prado, M.; da Silva, E.J.N.; Duque, T.M.; Zaia, A.A.; Ferraz, C.C.R.; de Almeida, J.F.A.; de Almeida Gomes, B.P.F. Antimicrobial and cytotoxic effects of phosphoric acid solution compared to other root canal irrigants. J. Appl. Oral Sci. 2015, 23, 158-163. [CrossRef] [PubMed] 
35. Tilg, H.; Grander, C. Microbiota and diabetes: An increasingly relevant association. Pol. Arch. Intern. Med. 2018, 128, 333-335. [CrossRef] [PubMed]

36. Godzien, J.; Ciborowski, M.; Martinez-Alcazar, M.P.; Samczuk, P.; Kretowski, A.; Barbas, C. Rapid and Reliable Identification of Phospholipids for Untargeted Metabolomics with LC-ESI-QTOF-MS/MS. J. Proteome Res. 2015, 14, 3204-3216. [CrossRef] [PubMed] 